\title{
Sutured Khovanov homology distinguishes braids from other tangles
}

\author{
J. Elisenda GRIGSBy AND Yi Ni
}

\begin{abstract}
We show that the sutured Khovanov homology of a balanced tangle in the product sutured manifold $D \times I$ has rank 1 if and only if the tangle is isotopic to a braid.
\end{abstract}

\section{Introduction}

In [11], Khovanov constructed a categorification of the Jones polynomial that assigns a bigraded abelian group to each link in $S^{3}$. Sutured Khovanov homology is a variant of Khovanov's construction that assigns

- to each link $\mathbb{L}$ in the product sutured manifold $A \times I$ (see Section 2.1) a triply-graded vector space $\operatorname{SKh}(\mathbb{L})$ over $\mathbb{F}:=\mathbb{Z} / 2 \mathbb{Z}[1,19]$, where $A=S^{1} \times[0,1]$ and $I=[0,1]$; and

- to each balanced, admissible tangle $T$ in the product sutured manifold $D \times I$ (see Section 2.2) a bigraded vector $\operatorname{space} \operatorname{SKh}(T)$ over $\mathbb{F}[6,13]$, where $D=D^{2}$.

Khovanov homology detects the unknot [14] and unlinks [3, 8], and the sutured annular Khovanov homology of braid closures detects the trivial braid [2]. In this note, we prove that the sutured Khovanov homology of balanced tangles distinguishes braids from other tangles.

Theorem 1.1. Let $T \subset D \times I$ be a balanced, admissible tangle. (See Section 2.2 for the definition.) Then $\operatorname{SKh}(T) \cong \mathbb{F}$ if and only if $T$ is isotopic to a braid in $D \times I$.

Theorem 1.1 is one of many results about the connection between Floer homology and Khovanov homology, starting with the work of Ozsváth and Szabó [18]. This theorem is an analog of the fact that sutured Floer homology detects product sutured manifolds $[10,17]$, which is also an ingredient in our proof. Other ingredients include a spectral sequence relating sutured 
Khovanov homology and sutured Floer homology [6], Meeks-Scott's theorem on finite group actions on product manifolds [15], and Kronheimer-Mrowka's theorem that Khovanov homology is an unknot detector [14].

Given a link $\mathbb{L} \subset A \times I$, the wrapping number of $\mathbb{L}$ is the minimal geometric intersection number of all links isotopic to $\mathbb{L}$ with the meridional disk of $A \times I$. Theorem 1.1 combined with the observations in [5] (see Proposition 2.4) imply:

Corollary 1.2. Let $\mathbb{L} \subset A \times I$ be a link with wrapping number $\omega$, then the group

$$
\operatorname{SKh}(\mathbb{L} ; \omega)=\bigoplus_{i, j} \operatorname{SKh}^{i}(\mathbb{L} ; j, \omega)
$$

is isomorphic to $\mathbb{F}$ if and only if $\mathbb{L}$ is isotopic to a closed braid in $A \times I$.

This corollary is an analog of the fact that knot Floer homology detects fibered knots.

\section{Preliminaries}

In this section, we will review the basics about sutured manifolds [4] and sutured Khovanov homology [1, 5, 6, 19].

Definition 2.1. A sutured manifold $(M, \gamma)$ is a compact, oriented 3-manifold $M$, a set $\gamma \subset \partial M$, and a choice of orientation on each component of $R(\gamma)=\partial M \backslash \operatorname{int}(\gamma)$ such that:

- $\gamma$ consists of pairwise disjoint annuli $A(\gamma)$ and tori $T(\gamma)$

- if we define $R_{+}(\gamma)$ (resp., $R_{-}(\gamma)$ ) to be the union of those components of $R(\gamma)$ whose normal vectors point out of (resp., into) $M$, then each component of $A(\gamma)$ is adjacent to a component of $R_{+}(\gamma)$ and a component of $R_{-}(\gamma)$.

As an example, let $S$ be a compact oriented surface, $M=S \times I, \gamma=$ $(\partial S) \times I, R_{-}(\gamma)=S \times\{0\}, R_{+}(\gamma)=S \times\{1\}$, then $(M, \gamma)$ is a sutured manifold. In this case, we say that $(M, \gamma)$ is a product sutured manifold.

Definition 2.2. [9, Definition 2.2] A balanced sutured manifold is a sutured manifold $(M, \gamma)$ satisfying

(1) $M$ has no closed components. 
(2) $T(\gamma)=\emptyset$

(3) Every component of $\partial M$ intersects $\gamma$ nontrivially.

(4) $\chi\left(R_{+}(\gamma)\right)=\chi\left(R_{-}(\gamma)\right)$.

If $(M, \gamma)$ is a balanced, sutured manifold, then $\operatorname{SFH}(M, \gamma)$ will denote its sutured Floer homology, as defined by Juhász in [9]. Whenever $\gamma$ is implicit (e.g., when $M$ is a product), we shall omit it from the notation.

We will be interested in Khovanov-type invariants for certain links and tangles in product sutured manifolds.

\subsection{Sutured Khovanov homology of links in $A \times I$}

Sutured annular Khovanov homology, originally defined in $[1,19]$ (see also [5]) associates with an oriented link $\mathbb{L}$ in the product sutured manifold $A \times I$ a triply-graded vector space

$$
\operatorname{SKh}(\mathbb{L})=\bigoplus_{i, j, k} \operatorname{SKh}^{i}(\mathbb{L} ; j, k),
$$

which is an invariant of the oriented isotopy class of $\mathbb{L} \subset A \times I$.

To define it, one chooses a diagram $\mathcal{D}_{\mathbb{L}}$ of $\mathbb{L}$ on $A \times\left\{\frac{1}{2}\right\}$. By filling in one boundary component of $A \times\left\{\frac{1}{2}\right\}$ with a disk marked with a basepoint $X$ at its center and the other boundary component with a disk marked with a basepoint at its center, one obtains a diagram on $S^{2}-\{X, O\}$. Ignoring the $X$ basepoint yields a diagram on $\mathbb{R}^{2}=S^{2}-\{O\}$ from which the ordinary bigraded Khovanov chain complex

$$
\operatorname{CKh}\left(\mathcal{D}_{\mathbb{L}}\right):=\bigoplus_{i, j} \operatorname{CKh}^{i}\left(\mathcal{D}_{\mathbb{L}} ; j\right)
$$

can be constructed from a cube of resolutions. Here, $i$ and $j$ are the homological and quantum gradings, respectively. The basepoint $X$ gives rise to a filtration on $\operatorname{CKh}\left(\mathcal{D}_{\mathbb{L}}\right)$, and $\operatorname{SKh}(\mathbb{L})$ is the homology of the associated graded object.

To define this filtration, choose an oriented arc from $X$ to $O$ missing all crossings of $\mathcal{D}_{\mathbb{L}}$. As described in [7, Section 4.2], the generators of $\operatorname{CKh}\left(\mathcal{D}_{\mathbb{L}}\right)$ are in one-to-one correspondence with oriented resolutions, where the counterclockwise orientation on each circle corresponds to the generator $v_{+}$. The " $k$ " grading of an oriented resolution is defined to be the algebraic intersection number of this resolution with our oriented arc. Roberts proves ([19, 
Lemmma 1]) that the Khovanov differential does not increase this extra grading.

One therefore obtains a bounded filtration,

$$
0 \subseteq \cdots \subseteq \mathcal{F}_{n-1}\left(\mathcal{D}_{\mathbb{L}}\right) \subseteq \mathcal{F}_{n}\left(\mathcal{D}_{\mathbb{L}}\right) \subseteq \mathcal{F}_{n+1}\left(\mathcal{D}_{\mathbb{L}}\right) \subseteq \cdots \subseteq \operatorname{CKh}\left(\mathcal{D}_{\mathbb{L}}\right)
$$

where $\mathcal{F}_{n}\left(\mathcal{D}_{\mathbb{L}}\right)$ is the subcomplex of $\operatorname{CKh}\left(\mathcal{D}_{\mathbb{L}}\right)$ generated by oriented resolutions with $k$ grading at most $n$. Let

$$
\mathcal{F}_{n}\left(\mathcal{D}_{\mathbb{L}} ; j\right)=\mathcal{F}_{n}\left(\mathcal{D}_{\mathbb{L}}\right) \cap \bigoplus_{i} \operatorname{CKh}^{i}\left(\mathcal{D}_{\mathbb{L}} ; j\right)
$$

The sutured annular Khovanov homology groups of $\mathbb{L}$ are defined to be

$$
\operatorname{SKh}^{i}(\mathbb{L} ; j, k):=H^{i}\left(\frac{\mathcal{F}_{k}\left(\mathcal{D}_{\mathbb{L}} ; j\right)}{\mathcal{F}_{k-1}\left(\mathcal{D}_{\mathbb{L}} ; j\right)}\right)
$$

It is an immediate consequence of the definitions that if $\mathbb{L}$ has wrapping number $\omega$, then $\operatorname{SKh}^{i}(\mathbb{L} ; j, k) \cong 0$ for $k \notin\{-\omega,-(\omega-2), \ldots, \omega-2, \omega\}$.

We shall denote by $\Sigma(A \times I, \mathbb{L})$ the sutured manifold obtained as the double cover of $A \times I$ branched along $\mathbb{L}$ (cf. [5, Remark 2.6]), where $\gamma$ is the cover of $(\partial A) \times I$, and $R_{+}$(resp., $R_{-}$) is the cover of $A \times\{1\}$ (resp., $A \times\{0\})$.

\subsection{Sutured Khovanov homology of balanced tangles in $D \times I$}

A tangle $T$ in the product sutured manifold $(D \times I, \gamma)$ is said to be admissible if $\partial T \cap \gamma=\emptyset$, and balanced if $|T \cap(D \times\{0\})|=|T \cap(D \times\{1\})|$. To make sense of tangle composition (stacking), we will fix an identification of $D$ with the standard unit disk in $\mathbb{C}$ and assume that $\partial T$ intersects both $D \times\{0\}$ and $D \times\{1\}$ along the real axis.

The sutured Khovanov homology of an admissible, balanced tangle in $D \times I$ was defined by Khovanov in [13, Section 5] in the course of constructing a categorification of the reduced $n$-colored Jones polynomial. An elaboration of Khovanov's construction is given in [6, Section 5], where it is also related to sutured Floer homology. We briefly recall the main points of the construction here.

Let $T \subset D \times I$ be a balanced, admissible tangle and choose a diagram $\mathcal{D}_{T}$ of $T$ on $[-1,1] \times I$. Then the sutured Khovanov homology of $T$, 
$\operatorname{SKh}(T)=\bigoplus_{i, j} \operatorname{SKh}^{i}(T ; j)$, is obtained as the homology of the complex,

$$
\operatorname{CKh}\left(\mathcal{D}_{T}\right):=\bigoplus_{i, j} \operatorname{CKh}^{i}\left(\mathcal{D}_{T} ; j\right)
$$

obtained as follows.

Number the $c$ crossings, and construct a Khovanov-type cube of resolutions whose vertices are in one-to-one correspondence with elements of $\{0,1\}^{c}$. Associated with each such $\mathcal{I} \in\{0,1\}^{c}$ is a complete resolution $R_{\mathcal{I}}$ with $a_{\mathcal{I}}$ closed components (circles) $T_{1}, \ldots, T_{a_{\mathcal{I}}}$ and $b_{\mathcal{I}}$ non-closed components (arcs) $T_{a_{\mathcal{I}}+1}, \ldots, T_{a_{\mathcal{I}}+b_{\mathcal{I}}}$. We say that $R_{\mathcal{I}}$ backtracks if the boundary of at least one of its non-closed components is contained in $[-1,1] \times\{1\}$. We now assign to the corresponding vertex in the cube of resolutions the vector space

$$
V\left(R_{\mathcal{I}}\right):= \begin{cases}0 & \text { if } R_{\mathcal{I}} \text { backtracks } \\ \Lambda^{*}\left(Z\left(R_{\mathcal{I}}\right)\right) & \text { otherwise }\end{cases}
$$

where

$$
Z\left(R_{\mathcal{I}}\right):=\frac{\operatorname{Span}_{\mathbb{F}}\left\{\left[T_{1}\right], \ldots,\left[T_{a_{\mathcal{I}}+b_{\mathcal{I}}}\right]\right\}}{\operatorname{Span}_{\mathbb{F}}\left(\left[T_{a_{\mathcal{I}}+1}\right], \ldots,\left[T_{a_{\mathcal{I}}+b_{\mathcal{I}}}\right]\right)}
$$

is the vector space formally generated by the closed components of $R_{\mathcal{I}}$, which for convenience we realize as a quotient space of the vector space formally generated by all components of $R_{\mathcal{I}}$.

As in ordinary Khovanov homology, if $\mathcal{I}^{\prime}$ is an immediate successor of $\mathcal{I}$ in the language of [18, Section 4] and [6, Section 4], then one obtains $R_{\mathcal{I}^{\prime}}$ from $R_{\mathcal{I}}$ by either merging two components $T_{i}$ and $T_{j}$ of $R_{\mathcal{I}}$ to form a component $T^{\prime}$ of $R_{\mathcal{I}}^{\prime}$ or splitting a single component $T$ of $R_{\mathcal{I}}$ into two components $T_{i}^{\prime}$ and $T_{j}^{\prime}$ of $R_{\mathcal{I}^{\prime}}$, and in both cases leaving all other components unchanged.

With the above understood, we now associate a map

$$
F_{R_{\mathcal{I}} \rightarrow R_{\mathcal{I}^{\prime}}}: V\left(R_{\mathcal{I}}\right) \rightarrow V\left(R_{\mathcal{I}^{\prime}}\right)
$$

to every pair of immediate successors as follows.

If at least one of $R_{\mathcal{I}}, R_{\mathcal{I}^{\prime}}$ backtracks, we define $F_{R_{\mathcal{I}} \rightarrow R_{\mathcal{I}^{\prime}}}:=0$.

Otherwise, $R_{\mathcal{I}} \rightarrow R_{\mathcal{I}^{\prime}}$ is either a merge or split cobordism involving either two closed components or one closed component and one non-backtracking arc. 
If $R_{\mathcal{I}} \rightarrow R_{\mathcal{I}^{\prime}}$ is a merge, we define $F_{R_{\mathcal{I}} \rightarrow R_{\mathcal{I}^{\prime}}}$ to be the composition

$$
V\left(R_{\mathcal{I}}\right) \stackrel{\pi}{\longrightarrow} \frac{V\left(R_{\mathcal{I}}\right)}{\left[T_{i}\right] \sim\left[T_{j}\right]} \stackrel{\alpha}{\longrightarrow} V\left(R_{\mathcal{I}}^{\prime}\right),
$$

where $\alpha$ is the isomorphism on exterior algebras induced by the isomorphism

$$
\frac{Z\left(R_{\mathcal{I}}\right)}{\left[T_{i}\right] \sim\left[T_{j}\right]} \cong Z\left(R_{\mathcal{I}^{\prime}}\right)
$$

identifying $\left[T_{i}\right]=\left[T_{j}\right]$ with $\left[T^{\prime}\right]$.

If $R_{\mathcal{I}} \rightarrow R_{\mathcal{I}^{\prime}}$ is a split, we define $F_{R_{\mathcal{I}} \rightarrow R_{\mathcal{I}^{\prime}}}$ to be the composition

$$
V\left(R_{\mathcal{I}}\right) \stackrel{\alpha^{-1}}{\longrightarrow} \frac{V\left(R_{\mathcal{I}}\right)}{\left[T_{i}^{\prime}\right] \sim\left[T_{j}^{\prime}\right]} \stackrel{\varphi}{\longrightarrow} V\left(R_{\mathcal{I}^{\prime}}\right),
$$

where $\varphi(a):=\left(\left[T_{i}^{\prime}\right]+\left[T_{j}^{\prime}\right]\right) \wedge \widetilde{a}$, and $\widetilde{a}$ is any lift of $a$ in $\pi^{-1}(a)$.

The image of $\theta \in V\left(R_{\mathcal{I}}\right)$ under the boundary map $\partial$ on the complex is now defined to be

$$
\partial(\theta):=\sum_{R_{\mathcal{I}^{\prime}}} F_{R_{\mathcal{I}} \rightarrow R_{\mathcal{I}^{\prime}}}(\theta)
$$

where the sum is taken over all immediate successors $\mathcal{I}^{\prime}$ to $\mathcal{I}$. Extend linearly.

Remark 2.3. If $T$ is an admissible $(n, n)$ tangle in $D \times I$ and $\mathcal{D}_{T}$ is a diagram of $T$, then we can alternatively associate with $T$ a left $H^{n}$-module, $\mathcal{F}\left(\mathcal{D}_{T}\right)$, as in [12], by viewing $T$ as a tangle with $2 n$ upper endpoints (cf. $\left[6\right.$, Remark 5.9]). The chain complex $\operatorname{CKh}\left(\mathcal{D}_{T}\right)$ may then be identified with $\overrightarrow{\mathbf{v}}_{-} \otimes_{H^{n}} \mathcal{F}\left(\mathcal{D}_{T}\right)$, where $\overrightarrow{\mathbf{v}}_{-}$is the right $H^{n}$ module constructed as follows. Let $b$ denote the fully-nested crossingless match on $2 n$ points; then $\overrightarrow{\mathbf{v}}_{-}$is the two-sided ideal of the $H^{n}$ module $\mathcal{F}(W(b) b)$ corresponding to the generator whose strands are all labeled with a $v_{-}$. Via the correspondence between oriented resolutions and Khovanov generators described in the previous section (cf. [7, Section 4.2]), we may then identify $\operatorname{CKh}\left(\mathcal{D}_{T}\right)$ as the quotient complex obtained from the ordinary Khovanov complex of the closure, $\widehat{\mathcal{D}}_{T}$, of $\mathcal{D}_{T}$ by the subcomplex generated by all generators with Roberts' " $k$ "grading less than $n$. This has the effect of setting to 0 any vertex associated with a backtracking resolution and treating the non-backtracking non-closed components of a resolution just as basepointed strands are treated in Khovanov's reduced theory.

Comparing the above description with the description of the sutured annular Khovanov invariant in the previous section, we have: 
Proposition 2.4 ([5, Theorem 3.1]). If $\mathbb{L} \subset A \times I$ is an oriented annular link with wrapping number $\omega$, and $T_{\theta}$ is the oriented, admissible balanced tangle obtained by decomposing $A \times I$ along a meridional disk $D_{\theta}$ for which $\left|\mathbb{L} \cap D_{\theta}\right|=\omega$,

$$
\operatorname{SKh}^{i}(\mathbb{L} ; j, \omega) \cong \operatorname{SKh}^{i}\left(T_{\theta} ; j\right) .
$$

Since all but one resolution of a braid backtracks, we have:

Proposition 2.5. If $T \subset D \times I$ is isotopic to a braid, then $\operatorname{SKh}(T) \cong \mathbb{F}$.

\section{Proof of the main theorem}

Definition 3.1. A tangle $T \subset D \times I$ is a string link if it consists of proper arcs, each of which has one end on $D \times\{0\}$ and the other end on $D \times\{1\}$.

As a consequence, a string link $T$ contains no closed components, and $T$ does not backtrack.

Lemma 3.2. Let $T \subset D \times I$ be a balanced, admissible tangle, then $\operatorname{dim}_{\mathbb{F}}$ $\operatorname{SKh}(T)$ is odd if and only if $T$ is a string link.

Proof. We observe that if two tangles $T_{+}, T_{-}$differ by a crossing change, then the corresponding chain complexes $\operatorname{CKh}\left(T_{+}\right)$and $\operatorname{CKh}\left(T_{-}\right)$have the same set of generators, thus the parities of the total dimensions of their homology are the same.

If a tangle $T$ has closed components, after crossing changes we can transform $T$ to a tangle $T^{\prime}$ with a diagram $\mathcal{D}^{\prime}$ containing a trivial loop. This loop persists in any complete resolution of $\mathcal{D}^{\prime}$, so it follows from the construction that the dimension of $\operatorname{CKh}\left(\mathcal{D}^{\prime}\right)$ is even, hence $\operatorname{dim}_{\mathbb{F}} \operatorname{SKh}(T)$ is even.

If $T$ backtracks, after crossing changes we can transform $T$ to a tangle $T^{\prime}$ with an arc which can be isotoped rel boundary into $D \times\{0\}$ or $D \times\{1\}$ without crossing other components. We can find a diagram $\mathcal{D}^{\prime}$ of $T^{\prime}$ such that any complete resolution of $\mathcal{D}^{\prime}$ backtracks. $\operatorname{So} \operatorname{CKh}\left(\mathcal{D}^{\prime}\right)=0$, and $\operatorname{dim}_{\mathbb{F}} \operatorname{SKh}(T)$ is even. 
If $T$ is a string link, after crossing changes we can transform $T$ to a braid $B$. By Proposition $2.5, \operatorname{SKh}(B) \cong \mathbb{F}$, $\operatorname{so~} \operatorname{dim}_{\mathbb{F}} \operatorname{SKh}(T)$ is odd.

Definition 3.3. A tangle $T \subset D \times I$ is split, if there exists a 3 -ball $B \subset$ $D \times I$, such that $L_{2}=T \cap B$ is a link and $L_{2} \neq T$. In this case, let $T_{1}=$ $T-L_{2}$, then we write $T=T_{1} \sqcup L_{2}$. We say $T$ is nonsplit if it is not split.

A tangle $T \subset D \times I$ is nonprime, if there exists a 3 -ball $B \subset D \times I$, such that $T_{2}=T \cap B$ is a $(1,1)$-tangle in $B$, and $T_{2}$ does not cobound a disk with any arc in $\partial B$. In this case, Let $T_{1} \subset D \times I$ be the tangle obtained by replacing $T_{2}$ with a trivial arc in $B$, and let $L_{2}$ be the link obtained from $T_{2}$ by connecting the two ends of $T_{2}$ by an $\operatorname{arc}$ in $\partial B$. We denote $T=T_{1} \# L_{2}$. We say $T$ is prime if there does not exist such a $B$.

Lemma 3.4. Let $(M, \gamma)$ be the sutured manifold which is the double branched cover of $D^{2} \times I$ branched along $T$. Then $M$ is irreducible if and only if $T$ is nonsplit and prime.

Proof. The conclusion follows from the Equivariant Sphere Theorem [16] by the same argument as in [8, Proposition 5.1].

Lemma 3.5. If $T=T_{1} \# L_{2}$ is a nonprime string link, then

$$
\operatorname{SKh}(T) \cong \operatorname{SKh}\left(T_{1}\right) \otimes K h_{r}\left(L_{2}\right) .
$$

In the above, $K h_{r}\left(L_{2}\right)$ denotes the reduced Khovanov homology of $L_{2}$.

Proof. We choose a diagram $\mathcal{D}_{T}$ of $T$ realized as the composition of diagrams $\mathcal{D}_{T_{1}}$ of $T_{1}$ and $\mathcal{D}_{L_{2}^{*, n}} L_{2}^{*, n}$, where $L_{2}^{*, n}$ is an $(n, n)$ tangle obtained from $L_{2}$ by removing a neighborhood of a point near the connected sum region and adjoining $n-1$ trivial strands as pictured in Figure 1.

Now we claim that

$$
\operatorname{CKh}\left(\mathcal{D}_{T}\right) \cong \operatorname{CKh}\left(\mathcal{D}_{T_{1}}\right) \otimes_{\mathbb{F}} \operatorname{CKh}\left(\mathcal{D}_{L_{2}^{*, n}}\right) .
$$

Since $\operatorname{CKh}\left(\mathcal{D}_{L_{2}^{*, n}}\right)$ is canonically chain isomorphic to $\operatorname{CKh}\left(\mathcal{D}_{L_{2}^{*, 1}}\right)$, and the homology of the latter complex is the reduced Khovanov homology of $L_{2}$ with $\mathbb{F}$ coefficients, the lemma will then follow from the Künneth theorem.

To see the claim, note first that each resolution $R$ of $\mathcal{D}_{T}$ is obtained by stacking a resolution $R_{1}$ of $\mathcal{D}_{T_{1}}$ and $R_{2}$ of $\mathcal{D}_{L_{2}^{*, n}}$.

Moreover:

- $R$ backtracks iff at least one of $R_{1}, R_{2}$ backtracks, and 


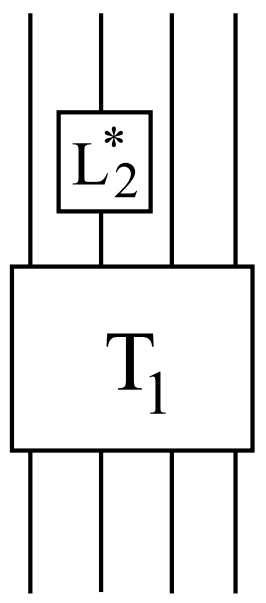

Figure 1: The tangle $T=T_{1} \# L_{2}$, realized as a composition of $T_{1}$ and $L_{2}^{*, n}$.

- If $R$ does not backtrack, then the number of closed components of $R$ is the sum of the number of closed components of $R_{1}$ and $R_{2}$.

Hence, the $\mathbb{F}$-vector space underlying the chain complex $\operatorname{CKh}\left(\mathcal{D}_{T}\right)$ is canonically isomorphic to $\operatorname{CKh}\left(\mathcal{D}_{T_{1}}\right) \otimes_{\mathbb{F}} \operatorname{CKh}\left(\mathcal{D}_{L_{2}^{*, n}}\right)$.

To verify that the boundary map $\partial_{T}$ on $\operatorname{CKh}\left(\mathcal{D}_{T}\right)$ agrees with the induced boundary map on the tensor product, i.e.,

$$
\partial_{T}=\partial_{T_{1}} \otimes \operatorname{Id}+\operatorname{Id} \otimes \partial_{L_{2}^{*, n}}
$$

it is sufficient to verify that the two maps agree on any decomposable generator $\theta=\theta_{1} \otimes \theta_{2}$ of $\operatorname{CKh}\left(\mathcal{D}_{T}\right)$ associated with a resolution $R=\left(R_{1}, R_{2}\right)$. We may further assume, without loss of generality, that $R$ does not backtrack.

By definition

$$
\partial_{T}(\theta)=\sum_{R^{\prime}=\left(R_{1}^{\prime}, R_{2}^{\prime}\right)} F_{R \rightarrow R^{\prime}}(\theta),
$$

where the sum above is taken over all immediate successors $R^{\prime}$ to $R$.

But if $R^{\prime}=\left(R_{1}^{\prime}, R_{2}^{\prime}\right)$ is an immediate successor of $R$, then either $R_{1}^{\prime}$ is an immediate successor of $R_{1}$ and $R_{2}^{\prime}=R_{2}$, or vice versa. Assume for definiteness that it is the former, the latter case being analogous.

If $R^{\prime}$ backtracks, then so does $R_{1}^{\prime}$, so:

$$
F_{R \rightarrow R^{\prime}}(\theta)=\left(F_{R_{1} \rightarrow R_{1}^{\prime}} \otimes \mathrm{Id}\right)\left(\theta_{1} \otimes \theta_{2}\right)=0
$$


If $R^{\prime}$ does not backtrack, then the saddle cobordism connecting $R_{1}$ to $R_{1}^{\prime}$ is a merge (resp., split) connecting either

- two closed components of $R_{1}$ (resp., of $R_{1}^{\prime}$ ); or

- one closed and one vertical component of $R_{1}$ (resp., of $R_{1}^{\prime}$ ).

In either case, we see that

$$
F_{R \rightarrow R^{\prime}}(\theta)=\left[F_{R_{1} \rightarrow R_{1}^{\prime}} \otimes \mathrm{Id}\right]\left(\theta_{1} \otimes \theta_{2}\right) .
$$

We conclude that

$$
\begin{aligned}
\partial_{T}(\theta) & =\left[\sum_{R^{\prime}=\left(R_{1}^{\prime}, R_{2}^{\prime}\right)} F_{R \rightarrow R^{\prime}}\right](\theta) \\
& =\left[\left(\sum_{R_{1}^{\prime}} F_{R_{1} \rightarrow R_{1}^{\prime}}\right) \otimes \mathrm{Id}+\mathrm{Id} \otimes\left(\sum_{R_{2}^{\prime}} F_{R_{2} \rightarrow R_{2}^{\prime}}\right)\right]\left(\theta_{1} \otimes \theta_{2}\right) \\
& =\left[\partial_{T_{1}} \otimes \mathrm{Id}+\mathrm{Id} \otimes \partial_{L_{2}^{*, n}}\right]\left(\theta_{1} \otimes \theta_{2}\right),
\end{aligned}
$$

as desired.

Proposition 3.6. Suppose that $T \subset D^{2} \times I$ is a balanced, admissible tangle. If the double branched cover of $D^{2} \times I$ branched along $T$ is a product sutured manifold, then $T$ is isotopic to a braid.

Proof. Let $\pi: F \times I \rightarrow D^{2} \times I$ be the double branched covering map, then the nontrivial deck transformation $\rho$ is an involution on $F \times I$ that preserves $F \times \partial I$ setwise. By Meeks-Scott [15, Theorem 8.1], $\rho$ is conjugate to a map preserving the product structure. ${ }^{1}$ In particular, $\pi^{-1}(T)$, being the set of fixed points of $\rho$, is homeomorphic to $P \times I \subset F \times I$ for some finite set $P \subset F$, via a homeomorphism of $F \times I$ which preserves $F \times \partial I$. It follows that $T$ is isotopic to a braid.

Proposition 3.7. A knot $K \subset S^{3}$ is the unknot if and only if $K h_{r}(K) \cong \mathbb{F}$.

Proof. This result is essentially a theorem of Kronheimer and Mrowka [14]. The original theorem of Kronheimer and Mrowka states that $K$ is the unknot if and only if $K h_{r}(K ; \mathbb{Z}) \cong \mathbb{Z}$, where the coefficients ring is $\mathbb{Z}$ while ours is

\footnotetext{
${ }^{1} \mathrm{~A}$ homeomorphism of $X \times Y$ preserves the product structure if it is the product of homeomorphisms of $X$ and $Y$.
} 
$\mathbb{F}$. However, the version with $\mathbb{F}$ coefficients easily follows from Kronheimer and Mrowka's argument. As shown in [14, Corollary 1.3],

$$
\operatorname{rank} K h_{r}(K ; \mathbb{Z}) \geq \operatorname{rank} I^{\natural}(K)
$$

Kronheimer and Mrowka proved that $\operatorname{rank} I^{\natural}(K)>1$ when $K$ is nontrivial. (See the paragraph after [14, Corollary 1.3].) So $\operatorname{rank} K h_{r}(K ; \mathbb{Z})>1$ when $K$ is nontrivial. It follows from the universal coefficients theorem that $\operatorname{dim}_{\mathbb{F}} K h_{r}(K ; \mathbb{F})>1$ when $K$ is nontrivial.

Proof of Theorem 1.1. By Lemma 3.2, if $\operatorname{SKh}(T) \cong \mathbb{F}$, then $T$ is a string link. In particular, $T$ has no closed components, hence $T$ must be nonsplit.

Since $T$ has no closed components, if $T$ is nonprime it must be the connected sum of a tangle with a knot (rather than a link). Suppose that $T=T_{1} \# K_{2}$, where $K_{2}$ is a knot. Then it follows from Lemma 3.5 that $K h_{r}\left(K_{2}\right) \cong \mathbb{F}$. Using Proposition 3.7, we conclude that $K_{2}$ is the unknot. Hence $T$ is prime.

Since $T$ is nonsplit and prime, Lemma 3.4 implies that $\Sigma(D \times I, T)$ is irreducible. Suppose that $\operatorname{SKh}(T) \cong \mathbb{F}$. By [6, Proposition 5.20], there is a spectral sequence whose $E^{2}$ term is $\operatorname{SKh}(T)$ and whose $E^{\infty}$ term is the sutured Floer homology group $S F H(\Sigma(D \times I, T))$. Hence $S F H(\Sigma(D \times$ $I, T)) \cong \mathbb{F}$. In $[10,17]$, it is shown that an irreducible balanced sutured manifold $(M, \gamma)$ is a product sutured manifold if and only if $S F H(M, \gamma) \cong \mathbb{F}$. Hence, $\Sigma(D \times I, T)$ is a product sutured manifold. Proposition 3.6 then implies that $T$ is isotopic to a braid.

\section{Acknowledgements}

The first author was partially supported by NSF grant numbers DMS0905848 and CAREER DMS-1151671. The second author was partially supported by NSF grant number DMS-1103976 and an Alfred P. Sloan Research Fellowship. We thank the anonymous referee for a number of valuable suggestions.

\section{References}

[1] M. Asaeda, J. Przytycki and A. Sikora, Categorification of the Kauffman bracket skein module of I-bundles over surfaces, Algebr. Geom. Topol. 4 (2004), 1177-1210. 
[2] J. Baldwin and J. Grigsby, Categorified invariants and the braid group, To appear in Proc. Amer. Math. Soc., 2012, available at: arXiv:math.GT/1212.2222.

[3] J. Batson and C. Seed, A link splitting spectral sequence in Khovanov homology 2013, available at: arXiv:math.GT/1303.6240.

[4] D. Gabai, Foliations and the topology of 3-manifolds, J. Differential Geom. 18(3) (1983), 445-503.

[5] J. Grigsby and S. Wehrli, Khovanov homology, sutured Floer homology, and annular links, Algebr. Geom. Topol. 10(4) (2010), 2009-2039.

[6] - On the colored Jones polynomial, sutured Floer homology, and knot Floer homology, Adv. Math. 223(6) (2010), 2114-2165.

[7] - On gradings in Khovanov homology and sutured Floer homology, in Topology and geometry in dimension three, 560, Contemporary Mathematics, 111-128, Amer. Math. Soc., Providence, RI, 2011.

[8] M. Hedden and Y. Ni, Manifolds with small Heegaard Floer ranks, Geom. Topol. 14(3) (2010), 1479-1501.

[9] A. Juhász, Holomorphic discs and sutured manifolds, Algebr. Geom. Topol. 6 (2006), 1429-1457.

[10] - Floer homology and surface decompositions, Geom. Topol. 12(1) (2008), 299-350.

[11] M. Khovanov, A categorification of the Jones polynomial, Duke Math. J. 101(3) (2000), 359-426.

[12] —, A functor-valued invariant of tangles, Algebr. Geom. Topol. 2 (2002), 665-741.

[13] - Categorifications of the colored Jones polynomial, J. Knot Theory Ramifications 14(1) (2005), 111-130.

[14] P. Kronheimer and T. Mrowka, Khovanov homology is an unknotdetector, Publ. Math. Inst. Hautes Études Sci. 113(1) (2011), 97-208.

[15] W. Meeks and P. Scott, Finite group actions on 3-manifolds, Invent. Math. 86(2) (1986), 287-346.

[16] W. Meeks, L. Simon and S. Yau, Embedded minimal surfaces, exotic spheres, and manifolds with positive Ricci curvature, Ann. Math. (2) 116(3) (1982), 621-659. 
Sutured Khovanov homology distinguishes braids from other tangles 1275

[17] Y. Ni, Knot Floer homology detects fibred knots, Invent. Math. 170(3) (2007), 577-608.

[18] P. Ozsváth and Z. Szabó, On the Heegaard Floer homology of branched double-covers, Adv. Math. 194(1) (2005), 1-33.

[19] L. Roberts, On knot Floer homology in double branched covers, 2007, available at: arXiv:math.GT/0706.0741.

Department of Mathematics, Boston College

CARNEY HALL

Chestnut Hill, MA 02467

USA

E-mail address: grigsbyj@bc.edu

Department of Mathematics, Caltech, MC 253-37

1200 E CALIFORNIA Blvd

PASADENA, CA 91125

USA

E-mail address: yini@caltech.edu

ReCEIVED June 11, 2013 
